\title{
Neue Pulvinsäure-Derivate aus Xerocomus chrysenteron (Bull. ex St. Amans) Quél. und Untersuchungen zur Frage des Vorkommens von Anthrachinonpigmenten bei Boletaceen
}

\author{
Wolfgang Steglich, Willibald Furtner und Axel Prox \\ Organisch-Chemisches Institut der Technischen Hochschule München \\ (Z. Naturforschg. 23 b, 1044-1050 [1968] ; eingegangen am 24. Februar 1968)
}

\begin{abstract}
Die sich an der Luft in Gegenwart von Oxydasen blau verfärbenden Pigmente von Boletus erythropus, B. calopus und Xerocomus chrysenteron gehören nicht dem Anthrachinon-1, sondern dem Pulvinsäuretyp an. In allen Fällen ist Variegatsäure $2^{4}$ die Hauptkomponente, daneben kommen ein neues blauendes Pigment, Xerocomsäure, und in X. chrysenteron noch Spuren von Atromentinsäure und einer Chlor-xerocomsäure vor. Die für Xerocomsäure massenspektrometrisch abgeleitete Struktur einer 3.4.4'-Trihydroxy-pulvinsäure (3 a bzw. $3 \mathrm{~b})$ wird synthetisch bewiesen. 1.2.4-Trihydroxy-anthrachinon-5-(oder 8)-carbonsäure ( 1 a oder 1 b) zeigt im Gegensatz zu den Angaben von Kögl und Deiss ${ }^{1}$ mit Oxydasen keine Blaufärbung, so daß sie als blauendes Prinzip der Boletaceen aus der Literatur zu streichen ist. Die Struktur der blauen Oxydationsprodukte wird diskutiert.
\end{abstract}

Boletol, das sich an der Luft in Gegenwart von Oxydasen tiefblau verfärbende Pigment verschiedener Pilze aus der Familie der Boletaceen, besitzt nach KöGL und DeIJs ${ }^{1}$ die Konstitution einer 1.2.4Trihydroxyanthrachinon-5-(oder 8)-carbonsäure (1a oder $1 \mathrm{~b}$ ).<smiles>O=C(O)c1cccc2c1C(=O)c1c(O)cc(O)c(O)c1C2=O</smiles><smiles>O=C(O)c1cccc2c1C(=O)c1c(O)cc(O)c(O)c1C2=O</smiles>

Die Verbindung ist biogenetisch interessant, da sie sich im Gegensatz zu anderen aus Basidiomyceten isolierten Anthrachinonen ${ }^{2}$ nicht vom Endocrocin ableiten läßt ${ }^{3}$. Uns erschien daher eine erneute Untersuchung ihrer Struktur wünschenswert.

\section{Isolierung der Farbstoffe}

Zur Isolierung der Farbstoffe wurden frisch gesammelte, zerkleinerte Fruchtkörper von Boletus erythropus Fr., B. calopus Fr. und Xerocomus chrysenteron (Bull. ex St. Amans) Quél. mit heißem Äthanol extrahiert. Nach Eindampfen der Lösungen wurde der Rückstand in heißem Wasser gelöst, fil-

1 F. Kögl u. W. B. Deiss, Liebigs Ann. Chem. 515, 10 [1935].

2 F. Kögl u. J. J. Postowsky, Liebigs Ann. Chem. 444, 1 [1925]. J. H. Birkinshaw u. R. Gourlay, Biochem. J. 80, 387 [1961]. W. Steglich u. V. Austel, Tetrahedron Letters [London] 1966, 3077. Inzwischen wurde auch Endocrocin aus Dermocybe sanguinea (Wulf. ex Fr.) Wünsche isoliert (W. STeglich u. W. Lösed, unveröffentlicht). triert und mehrfach mit Äther und Essigester ausgeschüttelt. Eindampfen der gelben Extrakte und Reinigung der Rohfarbstoffe an Aluminiumoxid lieferte aus Boletus erythropus $0,04 \%$, aus B. calopus $0,005 \%$ und aus Xerocomus chrysenteron 0,09\% eines roten Farbstoffs, der zum größten Teil aus der bereits von Edwards und Elsworthy ${ }^{4}$ aus Suillus variegatus (Sow. ex Fr.) O. Kuntze isolierten Variegatsäure 2 besteht.

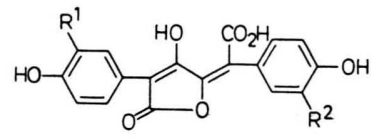

$$
\begin{array}{ll}
2, & \mathrm{R}^{1}=\mathrm{R}^{2}=\mathrm{OH} \\
3 \mathrm{a}, & \mathrm{R}^{1}=\mathrm{H}, \mathrm{R}^{2}=\mathrm{OH} \\
3 \mathrm{~b}, & \mathrm{R}^{1}=\mathrm{OH}, \mathrm{R}^{2}=\mathrm{H} \\
4, & \mathrm{R}^{1}=\mathrm{R}^{2}=\mathrm{H}
\end{array}
$$

Dünnschichtchromatographisch ist daneben noch ein zweiter Farbstoff nachweisbar, der bei den Boletus-Arten nur in Spuren, bei Xerocomus chrysenteron jedoch in größerer Menge vorkommt und Xerocomsäure genannt werden soll. Seine Reindarstellung gelang durch präparative Dünnschichtchromatographie, wobei gleichzeitig noch einige weitere

3 Vgl. z. B. S. Shibata, in: Beiträge zur Biochemie und Physiologie von Naturstoffen, Festschrift K. Мотнеs, VEB G. Fischer, Jena 1965, S. 451.

${ }^{4}$ R. L. Edwards u. G. C. Elsworthy, Chem. Commun. 1967, 373. 
in Spuren auftretende Farbstoffe abgetrennt wurden (Tab. 1).

\begin{tabular}{lll}
\hline \multicolumn{1}{c}{ Farbstoff } & $R_{f}{ }^{1}$ & \multicolumn{1}{c}{ Farbe } \\
\hline Variegatsäure & 0,04 & gelb, nach Trocknen grau \\
Xerocomsäure & 0,08 & gelb, nach Trocknen grau \\
Farbstoff C & 0,14 & gelb \\
Farbstoff D & 0,16 & gelb \\
Farbstoff E & 0,19 & $\begin{array}{l}\text { rotviolett, verblaßt beim } \\
\text { Trocknen }\end{array}$ \\
\hline
\end{tabular}

Tab. 1. Dünnschichtchromatographischer Vergleich der Farbstoffe aus Xerocomus chrysenteron. ${ }^{1}$ Kieselgel-Fertigplatten $\mathbf{F}_{254}$ von Merck, Darmstadt; Laufmittel: Benzol/Ameisensäuremethylester/Ameisensäure $(13: 5: 4)$.

\section{Konstitution und Synthese der Xerocomsäure}

Xerocomsäure zeigt in ihren spektroskopischen und chemischen Eigenschaften große Ähnlichkeit mit der Variegatsäure $2^{4}$. So treten im IR-Spektrum (KBr) Banden bei $3400-2300,1745,1685,1600$ und $1515 / \mathrm{cm}$ auf, die mit den entsprechenden von 2 weitgehend übereinstimmen. Die Lage der UV-Maxima ist bei beiden Verbindungen nahezu identisch (Tab. 2) und mit Kartoffespreßsaft oder

\begin{tabular}{ccl}
\hline \multicolumn{1}{c}{ Farbstoff } & \multicolumn{1}{c}{$\lambda_{\max }[\mathrm{nm}][\varepsilon]$} & $\begin{array}{c}\text { Lösungs- } \\
\text { mittel }\end{array}$ \\
\hline Variegatsäure & $275[10500], 415[7650]$ & Äthano] \\
& $259[12400], 381[6250]$ & Wasser \\
Xerocomsäure & $261[10700], 411[6700]$ & Äthanol \\
& $256[13800], 380[5700]$ & Wasser \\
\hline
\end{tabular}

Tab. 2. UV-Spektren von Variegatsäure und Xerocomsäure, aufgenommen mit einem UV-Gerät Beckman DK 25.

$\mathrm{K}_{3}\left[\mathrm{Fe}(\mathrm{CN})_{6}\right] / \mathrm{NaHCO}_{3}$ verfärbt sich die gelbe wäßrige Lösung tiefblau. Xerocomsäure liefert beim Erhitzen mit Acetanhydrid und einer Spur konz. Schwefelsäure ein gut kristallisierendes Triacetyllacton (Schmp. $221-223^{\circ} ;$ IR (KBr) : 1830 , $1775,1670,1610 / \mathrm{cm})$, das zu ihrer Charakterisierung geeignet ist.

Bei der Strukturermittlung der Xerocomsäure und der nur in Mengen um $1 \mathrm{mg}$ vorhandenen Nebenfarbstoffe C, D und E erwies sich die Massenspektrometrie als Methode der Wahl. Es sollen daher zunächst die hochaufgelösten Massenspektren der Variegatsäure und Xerocomsäure näher diskutiert werden, die in den Schemata 1 und 2 als sogenannte COHN-Diagramme ${ }^{5}$ wiedergegeben sind.

\begin{tabular}{|c|c|c|c|c|c|c|c|c|c|c|c|}
\hline Rel. & Masse & Elementar- & \multicolumn{9}{|c|}{ Elementardifferenzen } \\
\hline 100,0 & $\begin{array}{r}354,0376 \\
0,0000\end{array}$ & $\begin{array}{l}188 \quad 11 \\
100\end{array}$ & $\begin{array}{l}11 \\
10\end{array}$ & 22 & 33 & 44 & $\begin{array}{l}94 \\
50\end{array}$ & $\begin{array}{r}105 \\
50\end{array}$ & $\begin{array}{r}116 \\
50\end{array}$ & $\begin{array}{r}117 \\
70\end{array}$ & $\begin{array}{r}128 \\
70\end{array}$ \\
\hline 4,5 & $\begin{array}{r}326,0426 \\
-0,0005\end{array}$ & $\begin{array}{l}177 \\
100\end{array}$ & $1^{11}$ & 22 & 33 & $\begin{array}{l}83 \\
50\end{array}$ & $\begin{array}{l}94 \\
50\end{array}$ & $\begin{array}{r}105 \\
50\end{array}$ & $\begin{array}{r}106 \\
70\end{array}$ & $\begin{array}{r}117 \\
70\end{array}$ & \\
\hline 2,0 & $\begin{array}{r}325,0348 \\
-0,0006\end{array}$ & 177 & & & $\begin{array}{l}83 \\
40\end{array}$ & $\begin{array}{l}94 \\
40\end{array}$ & $\begin{array}{r}105 \\
40\end{array}$ & $\begin{array}{r}106 \\
60\end{array}$ & $\begin{array}{r}117 \\
60\end{array}$ & & \\
\hline 34,0 & $\begin{array}{r}298,0477 \\
0,0000\end{array}$ & $\begin{array}{l}166 \\
100\end{array}$ & $y^{22}$ & $\begin{array}{l}72 \\
50\end{array}$ & $\begin{array}{l}83 \\
50\end{array}$ & $\begin{array}{l}94 \\
50\end{array}$ & $\begin{array}{l}95 \\
70\end{array}$ & $\begin{array}{r}106 \\
70\end{array}$ & & & \\
\hline 1,5 & $\begin{array}{r}270,0528 \\
+0,0006\end{array}$ & $\begin{array}{l}155 \\
100\end{array}$ & $\begin{array}{l}61 \\
50\end{array}$ & $\begin{array}{l}72 \\
50\end{array}$ & $\begin{array}{l}83 \\
50\end{array}$ & $\begin{array}{l}84 \\
70\end{array}$ & $\begin{array}{l}95 \\
70\end{array}$ & & & & \\
\hline 32,0 & $\begin{array}{r}242,0579 \\
0,0000\end{array}$ & $\begin{array}{ll}144 & 50 \\
100 & 50\end{array}$ & $\begin{array}{l}61 \\
50\end{array}$ & $\begin{array}{l}72 \\
50\end{array}$ & $\begin{array}{l}73 \\
70\end{array}$ & $\begin{array}{l}84 \\
70\end{array}$ & & & & & \\
\hline 30,0 & $\begin{array}{r}177,0188 \\
0,0000\end{array}$ & $\begin{array}{l}94 \\
50\end{array}$ & 22 & $\begin{array}{l}23 \\
20\end{array}$ & $\begin{array}{l}34 \\
20\end{array}$ & & & & & & \\
\hline 70,0 & $\begin{array}{r}149,0239 \\
0,0000\end{array}$ & $\begin{array}{l}83 \\
50\end{array}$ & $\begin{array}{l}12 \\
20\end{array}$ & $\begin{array}{l}23 \\
20\end{array}$ & & & & & & & \\
\hline 55,0 & $\begin{array}{r}121,0290 \\
0,0000\end{array}$ & $\begin{array}{ll}72 & 1 \\
50 & 20\end{array}$ & $\begin{array}{r}12 \\
20\end{array}$ & & & & & & & & \\
\hline 3,0 & $\begin{array}{r}103,0184 \\
+0,0004\end{array}$ & $\begin{array}{l}71 \\
30\end{array}$ & & & & & & & & & \\
\hline 30,0 & $\begin{array}{r}75,0235 \\
+0,0004\end{array}$ & $\begin{array}{l}60^{\prime} \\
30\end{array}$ & & & & & & & & & \\
\hline
\end{tabular}

Schema 1. COHN-Diagramm des Variegatsäurelaktons.

5 W. Kosbahn u. A. Prox, Organic Mass Spectrometry, in Vorbereitung. 


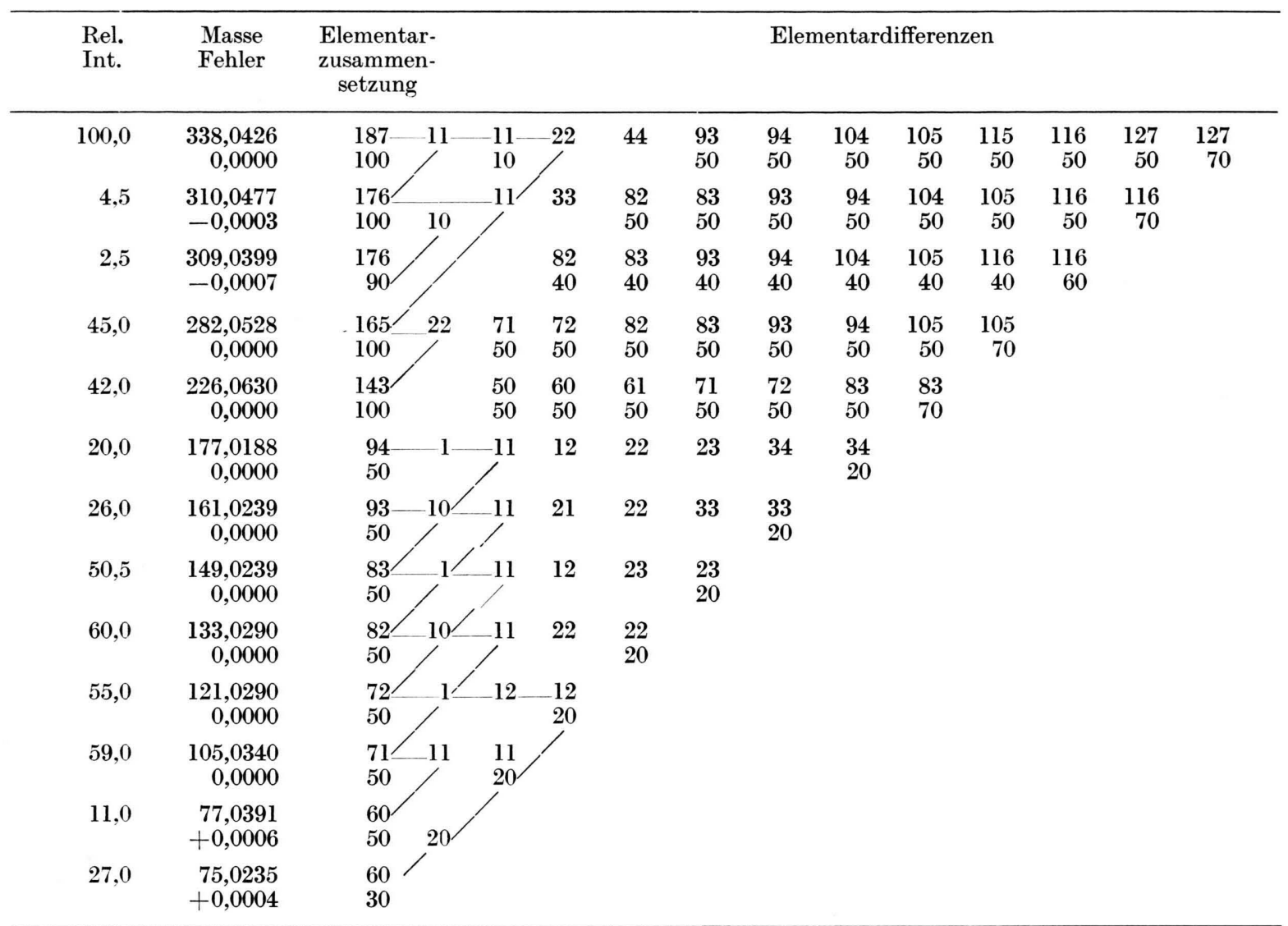

Schema 2. COHN-Diagramm des Xerocomsäurelaktons.

Die Diagramme sind eine Weiterentwicklung des früher vorgeschlagenen Differenzschemas ${ }^{6}$. Sie enthalten von links Angaben über die Intensität, die theoretische Masse mit den darunter angegebenen Fehlern der Messungen in ME und die elementare Zusammensetzung der Ionen in Form von zwei dreistelligen Zahlen, die untereinander notiert sind. Beispielsweise erscheint für das Ion $m / e 354,0376$ (Schema 1) seine elementare Zusammensetzung als ${ }_{100}^{188}$. Darin geben die beiden ersten Stellen der oberen Ziffer die Zahl der im Ion enthaltenen C-Atome, die dritte Stelle die Anzahl der O-Atome an. Entsprechend bedeuten die beiden ersten Stellen der unteren Ziffer die Zahl der H-Atome und die dritte Stelle die An7ahl der N-Atome. Damit ergibt sich die Anordnung $\underset{\mathrm{HN}}{\text { co }}$, so daß die Gruppe ${ }_{100}^{188}$ einem Ion der Zusammensetzung $\mathrm{C}_{18} \mathrm{H}_{10} \mathrm{O}_{8}$ entspricht. Alle aus dem Massenspektrum vermessenen Ionen werden mit abnehmender Masse untereinander aufgezeichnet. Rechts von der elementaren Zusammensetzung werden mit den gleichen Zahlengruppen alle Elementardifferenzen zu solchen massenniedrigeren Ionen notiert, bei denen sich für die jeweils enthaltenen Elemente der Wert null oder eine positive ganze Zahl ergibt. Wird einer dieser Werte negativ, dann kann das massenniedrigere Ion auf keinen Fall aus dem höheren entstanden sein, und es erscheint eine Leerstelle. Dieses Diagramm wird aus den eingegebenen hochaufgelösten Daten von einem Computer berechnet. Metastabile

6 F. Weygand, A. Prox, H. H. Fessel u. K. K. Sun, Z. Naturforschg. 20 b, 1169 [1965].
Zerfälle lassen sich durch Verbinden der betreffenden Ionen mit der Zahlengruppe für ihre Elementardifferenzen einfach und übersichtlich wiedergeben.

Als massenhöchstes Ion erscheint in beiden Spektren das Molekularion des entsprechenden Lactons (A, Schema 3), das sich durch Wasserabspaltung aus der Hydroxysäure im Direkteinlaßsystem bildet. Danach unterscheidet sich Xerocomsäure von 2 nur durch das Fehlen eines Sauerstoffatoms.

In ihrem Zerfallsverhalten zeigen beide Verbindungen große Ähnlichkeit mit dem kürzlich von Letcher und EGGERs ${ }^{7}$ untersuchten Pulvinsäurelacton. Aus der Berechnung der metastabilen Ionen ergeben sich für 2 zwei Reihen aufeinanderfolgender Zerfälle, die in das COHN-Diagramm eingezeichnet sind. Die erste Folge leitet sich vom Molekularion $m / e 354$ ab. Sie umfaßt vier nacheinander erfolgende CO-Eliminierungen. Charakteristische metastabile Banden zeigen, daß dabei überwiegend jeweils zwei $\mathrm{CO}$, möglicherweise als $\mathrm{C}_{2} \mathrm{O}_{2}$, nacheinander eliminiert werden. Die zweite Reihe von metastabilen Zer- 
fällen geht vom Ion $m / e 177$ aus und umschließt zwei CO-Eliminierungen und die nachfolgende $\mathrm{Ab}$ spaltung von $\mathrm{CH}_{2} \mathrm{O}_{2}$. Möglicherweise erfolgt der letzte Zerfall in zwei Schritten, wobei für die zunächst eintretende Wasserabspaltung gleichfalls eine metastabile Bande beobachtet wird.

Im Schema 3 ist eine formelmäßige Deutung der beobachteten Zerfälle wiedergegeben. In Übereinstimmung mit den genannten Autoren ${ }^{7}$ wird primär eine Umlagerung des Lactonsystems unter Elektronenbeschuß angenommen. Das dabei entstehende Ion B kann aus seiner cyclischen Form $\mathrm{C}$ heraus sowohl durch schrittweise Abspaltung der vier COGruppen als auch durch doppelte konsekutive Eliminierung von $\mathrm{C}_{2} \mathrm{O}_{2}$ zerfallen. Der Bruch der $\mathrm{C}-\mathrm{C}$ Bindung zwischen den beiden Carboxylgruppen in B erklärt zwanglos den Zerfall des $\mathbf{M}^{\oplus}$-Ions in zwei Hälften. Die dabei gebildeten Ionen mit gerader Elektronenzahl lassen sich im Massenspektrum an ihrer ungeraden Massenzahl erkennen. Aus $2\left(\mathrm{R}^{1}=\right.$ $\mathrm{R}^{2}=\mathrm{OH}$ in Schema 3) entsteht dabei das Ion $m / e$ 177, das wie das entsprechende Fragmention des Pulvinsäurelactons ${ }^{7}$ zunächst unter doppelter $\mathrm{CO}$ Abspaltung weiterzerfällt. Der anschließende Verlust von $\mathrm{CH}_{2} \mathrm{O}_{2}$ ist offensichtlich für den 3.4-dihydroxylierten Phenylkern charakteristisch.

Auch im Massenspektrum des Xerocomsäurelactons (Schema 2) wird der typische Zerfall des Lactonsystems ausgehend vom $\mathrm{M}^{\oplus}$-Ion $\mathrm{m} / \mathrm{e} 338 \mathrm{beob}$ achtet. Dabei läßt sich neben der doppelten konsekutiven Eliminierung von zwei CO nur die schrittweise Eliminierung von zwei einzelnen CO-Molekeln nachweisen, ganz in Analogie zum Pulvinsäurelacton ${ }^{7}$. Das Fehlen eines Sauerstoffatoms führt gegenüber 2 zu einer massenspektrometrischen Verschiebung der entsprechenden Ionen $\left(\mathrm{R}^{\mathbf{1}}=\mathrm{H}, \mathrm{R}^{\mathbf{1}}=\mathrm{OH}\right)$ um 16 ME. Die Art der Verteilung der drei Sauerstoffatome auf die beiden Phenylkerne geht eindeutig aus den weiteren Fragmentionen hervor. Im Gegensatz zu 2 werden im Massenspektrum des Xerocomsäurelactons zwei weitere Folgen von metastabilen Zerfällen gefunden. Sie leiten sich von den Ionen $m / e 177$ und 161 ab. Da der Zerfall von $m / e 177$ in allen Einzelheiten dem des gleichen Ions im Spektrum von 2 entspricht, kann geschlossen werden, daß einer der beiden Phenylringe der Xerocomsäure zwei Hydroxygruppen in der gleichen Anordnung

7 R. M. Letcher u. S. H. EgGers, Tetrahedron Letters [London] 1967, 3541 .

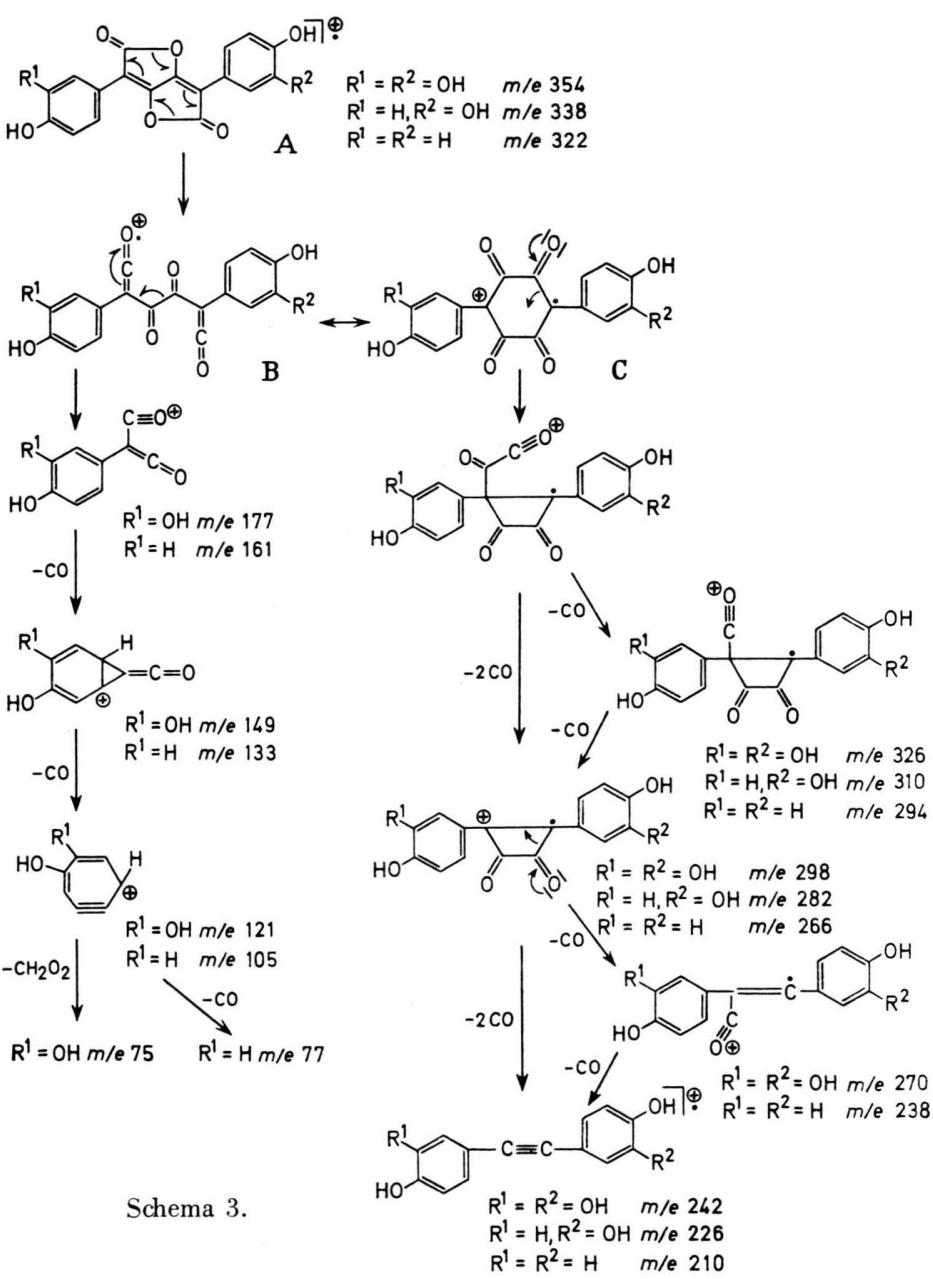

enthält wie die Variegatsäure. Das Ion $m / e 161$ besitzt ein Sauerstoffatom weniger und verliert nacheinander drei CO.

Xerocomsäure ist demnach eine 3.4.x'-Trihydroxy-pulvinsäure, wobei die einzelne Hydroxygruppe aus biogenetischen Gründen in 4'-Stellung stehen sollte. Da eine Isomerisierung unter den Isolierungsbedingungen nicht auszuschließen ist, liegt wahrscheinlich ein Gemisch von $3 \mathrm{a}$ und $3 \mathrm{~b}$ vor.

Die vorgeschlagene Struktur wurde durch eine Synthese bewiesen, bei der in Anlehnung an Asano und Kameda ${ }^{8}$ [3.4-Dimethoxyphenyl]-cyan-brenztraubensäure-äthylester ${ }^{9}$ mit $p$-Methoxy-benzylcyanid zum Ketipinsäure-Derivat 5 kondensiert wurde. Verseifung mit $\mathrm{HBr} /$ Eisessig und anschließende Ace-

8 M. Asano u. Y. Kameda, Ber. dtsch. chem. Ges. 67, 1522 [1934].

9 R. K. Hill, J. org. Chemistry 26, 4745 [1961]. 
tylierung lieferte Triacetylxerocomsäurelacton 6 , in jeder Beziehung identisch mit der aus dem Naturprodukt erhaltenen Verbindung. 6 kann durch saure Hydrolyse in Xerocomsäure übergeführt werden.

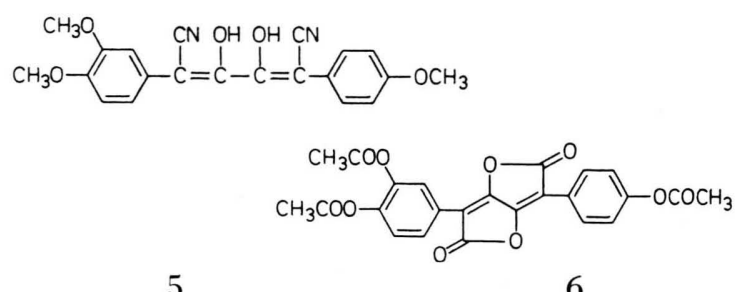

\section{Zur Struktur der Nebenfarbstoffe $C, D$ und $E$}

Der nur in einer Menge von etwa $1 \mathrm{mg}$ isolierte Farbstoff $\mathrm{C}$ besitzt in wäßriger Lösung ein UV-Maximum bei $370 \mathrm{~nm}$. Nach Zugabe von Kartoffelpreßsaft tritt keine Blaufärbung auf. Das Massenspektrum zeigt wiederum das für Pulvinsäure-Derivate charakteristische Bild. Ausgehend vom Molekularion $m / e 322$ treten die in Schema $3\left(\mathrm{R}^{1}=\mathrm{R}^{2}=\mathrm{H}\right)$ eingezeichneten Zerfälle $\mathrm{m} / \mathrm{e} 294 \rightarrow 238 \rightarrow 210$ und $161 \rightarrow 133 \rightarrow 105 \rightarrow 77$ auf, wobei der letztere völlig dem der Xerocomsäure entspricht. Es kann daher kein Zweifel bestehen, daß der Farbstoff C mit Atromentinsäure $4^{10}$ identisch ist, die damit erstmals in der Natur nachgewiesen wurde. Das gleichzeitige Vorkommen der drei nahe verwandten PulvinsäureDerivate in Xerocomus chrysenteron deutet darauf hin, daß Variegatsäure biosynthetisch über Atromentin, Atromentinsäure und Xerocomsäure entsteht.

Auch der Farbstoff D erwies sich nach dem Massenspektrum als Pulvinsäure-Derivat. Die charakteristische Isotopenverteilung und das Zerfallsverhalten sprechen für eine Chlor-xerocomsäure $\left(\mathrm{M}^{\oplus}=\right.$ 373/375), wobei das Chloratom nach den Fragmentionen $195 / 197 \rightarrow 167 / 169 \rightarrow 149 / 151$ an dem Phenylring sitzt, der nur eine Hydroxygruppe trägt. Da die Substanz nur in sehr geringer Menge vorliegt, war eine weitere Charakterisierung nicht möglich.

Der in organischen Solventien mit tiefroter Farbe lösliche Farbstoff $\mathrm{E}$ ist wahrscheinlich ein Artefakt, das während der Aufarbeitung der Pigmente durch Oxydation von 2 entsteht. Es besitzt nach dem hochaufgelösten Massenspektrum die Summenformel $\mathrm{C}_{18} \mathrm{H}_{10} \mathrm{O}_{9}$.

10 F. Kögl u. H. Becker, Liebigs Ann. Chem. 465, 211 [1928].
Zur Struktur der bei der Oxydation von Variegatund Xerocomsäure entstehenden blauen Farbstoffe

Die aus 2, 3 und synthetischer 3.4-Dihydroxypulvinsäure durch Einwirkung von Oxydasen oder $\mathrm{K}_{3}\left[\mathrm{Fe}(\mathrm{CN})_{6}\right] / \mathrm{NaHCO}_{3}$ erhaltenen tiefblauen wäßrigen Lösungen zeigen in ihren Absorptionsspektren (Tab.3) eine große Ähnlichkeit mit dem kürzlich von WANZLICK ${ }^{11}$ beschriebenen Anion 7 (Abb. 1).

\begin{tabular}{|c|c|c|}
\hline $\begin{array}{l}\text { Pulvinsäure- } \\
\text { Derivat }\end{array}$ & $\begin{array}{l}\text { Blauer } \\
\text { Farbstoff }\end{array}$ & $\lambda_{\max }[\mathrm{nm}][\varepsilon]$ \\
\hline $\begin{array}{l}\text { 3.4-Dihydroxy- } \\
\text { pulvinsäure }\end{array}$ & $8 \mathrm{a}$ & $\begin{array}{l}399[15500], 415 \\
\text { (Schulter), } 589[6200]\end{array}$ \\
\hline $\begin{array}{l}\text { Xerocomsäure } \\
\text { (3a bzw. } 3 \text { b) }\end{array}$ & $8 \mathrm{~b}$ & $\begin{array}{l}376 \text { (Schulter), } \\
394 \text { [14 100], } 413 \\
\text { (Schulter), } 604 \text { [6500] }\end{array}$ \\
\hline Variegatsäure (2) & $8 \mathrm{c}$ & $\begin{array}{l}371[11200], 392,415 \\
\text { (Schultern), } 595^{1}[7000]\end{array}$ \\
\hline
\end{tabular}

Tab. 3. Sichtbare und UV-Spektren der bei der Oxydation von 2, 3 und 3.4-Dihydroxy-pulvinsäure mit $\mathrm{K}_{3}\left[\mathrm{Fe}(\mathrm{CN})_{6}\right] /$ $\mathrm{NaHCO}_{3}$ in Wasser entstehenden blauen Lösungen. ${ }^{1} \mathrm{Bei}$ höherer Konzentration 605 nm, wie l. c. ${ }^{4}$.

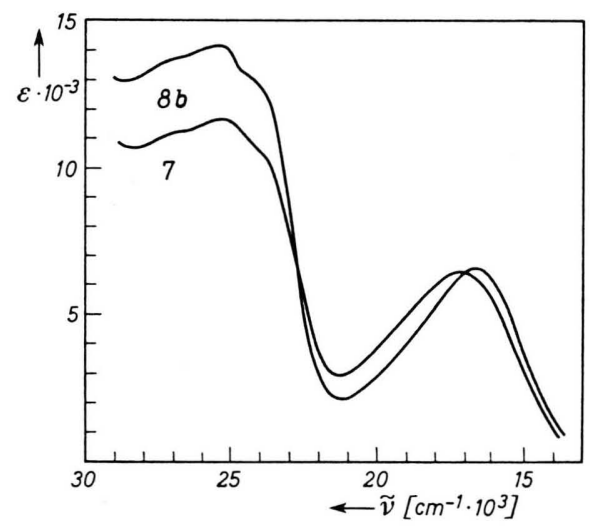

Abb. 1. Sichtbares und UV-Spektrum der Anionen 7 und 8 b in wäßriger Lösung. Die Extinktionskoeffizienten gelten nur für $8 \mathrm{~b}$, für 7 wurden nur die Extinktionen bestimmt.

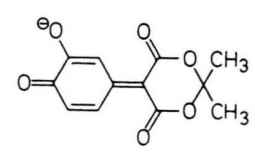

7

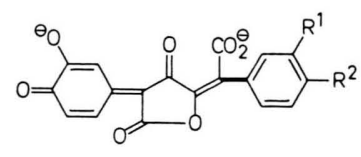

8

$$
\begin{aligned}
8 \mathrm{a}, & \mathrm{R}^{1}=\mathrm{R}^{2}=\mathrm{H} \\
\mathrm{b}, & \mathrm{R}^{1}=\mathrm{H}, \mathrm{R}^{2}=\mathrm{OH} \\
\mathrm{c}, & \mathrm{R}^{1}=\mathrm{R}^{2}=\mathrm{OH}
\end{aligned}
$$

11 H.-W. W Anzlick $_{\text {u. R. Gritzky, zitiert bei H.-W. W }}$ ANzlick, Angew. Chem. 76, 313 [1964]. 
7 entsteht aus Brenzkatechin und Meldrumsäure in gepufferter wäßriger Lösung durch Oxydation mit $\mathrm{K}_{3}\left[\mathrm{Fe}(\mathrm{CN})_{6}\right]^{11}$ oder Kartoffelpreßsaft. Die blauen Oxydationsprodukte der Pulvinsäuren sind demnach als Anionen der Chinonmethide $8 \mathrm{zu}$ formulieren. Die Struktur 8 erklärt das Ausbleiben der Farbreaktion bei Atromentinsäure $4^{4}$.

\section{Zur Frage des Vorkommens von Anthrachinon- Pigmenten bei Boletaceen}

Trotz sorgfältiger Untersuchung aller Dünnschichtfraktionen konnten wir in keiner der genannten Boletaceen „Boletol“ ( 1 a oder $1 \mathrm{~b}$ ) auffinden. Das synthetische Gemisch von 1 a und $1 b^{12}$ ist unter unseren Isolierungsbedingungen stabil und läßt sich leicht neben den in Tab. 1 aufgeführten Pigmenten nachweisen $\left(R_{f}=0,23\right)$. Im Gegensatz zu den Angaben von KögL und Deiss ${ }^{1,12}$ konnten wir die Trihydroxy-anthrachinoncarbonsäuren weder mit Kartoffelpreßsaft noch mit Oxydationsmitteln wie Bleitetraacetat oder $\mathrm{K}_{3}\left[\mathrm{Fe}(\mathrm{CN})_{6}\right] / \mathrm{NaHCO}_{3}$ zu einem blauen Farbstoff oxydieren.

Damit sind Anthrachinon-Derivate als blauende Prinzipien der Boletaceen aus der Literatur zu streichen. Die charakteristische Blaufärbung mit Oxydasen wird bei allen bisher untersuchten Arten der Gattungen Boletus, Xerocomus und Suillus ${ }^{4}$ allein durch Pulvinsäure-Derivate verursacht. Auch das von Gabriel ${ }^{13}$ in 22 Arten der Gattungen Boletus, Phylloporus, Xerocomus, Boletinus, Suillus und Gyrodon chromatographisch nachgewiesene „Boletol" ist nach den $R_{f}$-Werten und dem UV-Spektrum sicher mit 2 identisch. Aus gleichen Gründen dürfte es sich bei dem in Boletus erythropus, Xerocomus parasiticus (Bull. ex Fr.) Quél., Suillus placidus (Bon.) Sing. und S. piperatus (Bull. ex Fr.) O. Kuntze vorkommenden „Pseudoboletol“ um Xerocomsäure handeln.

Erwähnt sei, daß Bовнм ${ }^{14}$ bereits 1885 den blauenden Farbstoff von Boletus luridus untersuchte und dabei ein Präparat isolierte, das er Luridussäure nannte. Mehrere seiner Angaben für Luridussäure treffen auch für Variegatsäure zu, so der Farbumschlag der gelben wäßrigen Lösung über grün nach tiefblau bei Zugabe von Soda, die violette $\mathrm{FeCl}_{3}$-Reaktion und die Farbreaktionen mit konz. $\mathrm{HNO}_{3}$ (kirschrot) und Jodtinktur (blau). Beide

12 F. Kögl u. W. B. Deiss, Liebigs Ann. Chem. 515, 23 [1935]. 13 M. Gabriel, Thèse, Lyon 1965.
Säuren werden mit Bleiacetat als orangebraune Niederschläge gefällt, die sich beim Trocknen an der Luft schmutzig verfärben. Danach ist BoEнms Luridussäure zweifellos mit einer verunreinigten Variegatsäure identisch.

\section{Beschreibung der Versuche}

Die Aufnahme der Massenspektren erfolgte mit einem Massenspektrometer MS 9 von AEI über ein Direkteinlaßsystem bei $70 \mathrm{eV}$. Die hochauflösenden Messungen wurden bei einer Auflösung von 20000 (10\% Taldefinition) ausgeführt. Die metastabilen Ionen wurden mit Hilfe eines Computerprogramms berechnet, wobei die Abweichung stets kleiner als $\pm 0,2 \%$ der Masse der betreffenden metastabilen Bande war. Die IR-Spektren wurden mit dem Infrachord von PerkinElmer, die UV-Spektren mit dem DK 25 von Beckman aufgenommen.

$$
\text { Isolierung der Farbstoffe }
$$

$5,82 \mathrm{~kg}$ frisch gesammelter Fruchtkörper von Xerocomus chrysenteron wurden in $9 l$ heißem Äthanol mit einem Vibrationsstab zerkleinert. Die Lösung wurde abgesaugt und der Filterrückstand gut mit Äthanol gewaschen. Eindampfen des orangegelben Filtrats i. Vak. lieferte einen Rohextrakt, der mit $600 \mathrm{ml}$ Wasser aufgekocht, heiß filtriert und nach dem Abkühlen dreimal mit je $400 \mathrm{ml}$ Äther ausgeschüttelt wurde. Nun wurde mit $\mathrm{HCl}$ angesäuert und dreimal mit je $500 \mathrm{ml}$ Essigester extrahiert. Nach dem Dünnschichtchromatogramm enthielten die Essigesterextrakte neben 2 nur Spuren Xerocomsäure, die Ätherextrakte neben 2 die in Tab. 1 aufgeführten Farbstoffe.

Die Ätherextrakte wurden über $\mathrm{Na}_{2} \mathrm{SO}_{4}$ getrocknet, eingedampft und auf mit Kieselgel $\mathrm{G}$ belegten Platten im System Benzol/Ameisensäureäthylester/Ameisensäure $(13: 5: 4)$ chromatographiert. Die unterste Zone bestand aus Variegatsäure, gefolgt von Xerocomsäure und schwachen Zonen der Farbstoffe C, D und E. Die Zonen wurden sofort nach Herausnehmen der Platten abgekratzt und noch feucht zwischen verd. $\mathrm{HCl}$ und Essigester verteilt. Eindampfen der mit Wasser gewaschenen, getrockneten Extrakte lieferte insgesamt $220 \mathrm{mg} \mathrm{2,} 50 \mathrm{mg}$ Xerocomsäure, jeweils etwa $1 \mathrm{mg}$ der Farbstoffe C und D und etwa $10 \mathrm{mg}$ Farbstoff E. Die Xerocomsäure schmolz im geschlossenen Rohr bei $295^{\circ}$ (Zers.).

Die vereinigten Essigesterextrakte wurden nach dem Trocknen auf ca. $50 \mathrm{ml}$ eingeengt und auf eine kurze $\mathrm{Al}_{2} \mathrm{O}_{3}$-Säule gegeben. Der Rohfarbstoff wurde nach Waschen mit ca. $5 l \mathrm{SO}_{2}$-haltigem Essigester und $\mathrm{Me}$ thanol/konz. HCl $(100: 1)$ eluiert. Eindampfen des Eluates i. Vak., Lösen des Rückstandes in verd. HCl und erneute Extraktion mit Essigester lieferte nach dem

14 R. Boenм, Naunyn-Schmiedebergs Arch. exp. Pathol. Pharmakol. 19, 60 [1885]. 
Eindampfen $220 \mathrm{mg}$ Variegatsäure, die nur Spuren von Xerocomsäure enthielt. Schmp. $302^{\circ}$ (Zers., im geschlossenen Rohr), Lit. l.c. ${ }^{4}>320^{\circ}$, identisch mit einem nach 1. c. ${ }^{4}$ synthetisierten authentischen Präparat. Zur Charakterisierung wurde noch das Tetraacetylvariegatsäurelacton ${ }^{4}$ dargestellt.

Nach dem gleichen Aufarbeitungsgang wurden aus $260 \mathrm{~g}$ Boletus erythropus $98 \mathrm{mg} 2$ und $2 \mathrm{mg}$ Xerocomsäure isoliert. 440 g. B. calopus lieferten $25 \mathrm{mg}$ Variegatsäure.

$$
\text { Triacetyl-xerocom säurelacton }
$$

$25 \mathrm{mg}$ Xerocomsäure wurden mit $1 \mathrm{ml}$ Acetanhydrid und einer Spur konz. Schwefelsäure 5 Min. unter Rückfluß gekocht. Nach dem Abkühlen schieden sich zitronengelbe Nadeln ab, die noch zweimal aus Eisessig umkristallisiert wurden. Schmp. $221-223^{\circ}$, nach Sintern ab $213^{\circ}$. UV-Spektrum: $\lambda_{\max }=378 \mathrm{~nm} \quad(\varepsilon=33500$, in Aceton); IR (KBr): 1830, 1775, 1670, 1610, 1510, $1370,1205 / \mathrm{cm}$.

$$
\begin{array}{lllll}
\mathrm{C}_{24} \mathrm{H}_{16} \mathrm{O}_{10}(464,4) & \text { Ber. } & \text { C } 62,07 & \text { H } 3,47, \\
& \text { Gef. } & \text { C } 61,83 & \text { H } 3,38 .
\end{array}
$$

Synthese der Xerocomsäure

\section{a) 3.4-Dimethoxyphenyl-4'-methoxyphenyl-ketipin- säure-dinitril

4,4 g $p$-Methoxy-benzylcyanid wurden in einer Lösung von $1,2 \mathrm{~g}$ Natrium in $20 \mathrm{ml}$ absol. Äthanol gelöst. Nach Zusatz von 7,8 g [3.4-Dimethoxyphenyl]-cyanbrenztraubensäureäthylester ${ }^{9}$ wurde gut umgeschüttelt und 90 Min. auf dem Wasserbad erwärmt. Zum abgekühlten Reaktionsgemisch gab man bis zur klaren Lösung ca. $50 \mathrm{ml}$ Wasser, säuerte mit 3,5 ml Eisessig an und verdünnte nochmals mit $200 \mathrm{ml}$ Wasser. Der orangefarbene, voluminöse Niederschlag wurde abgesaugt und aus Eisessig umkristallisiert. Ausbeute 8,5 g (88\% d. Th.), Schmp. $221-222^{\circ}$ (Zers., sintert ab $\left.215^{\circ}\right)$. UV-Spektrum: $\lambda_{\max }=348 \mathrm{~nm} \quad(\varepsilon=21200)$, $418 \mathrm{~nm}$ (Schulter, $\varepsilon=4600$ ).

$\mathrm{C}_{21} \mathrm{H}_{18} \mathrm{~N}_{2} \mathrm{O}_{5}(378,4)$ Ber. C 66,65 H 4,80 N 7,41, Gef. C 66,15 H 4,81 N 6,85. b) Triacetyl-xerocomsäurelacton (6)

2 g 5 wurden in $40 \mathrm{ml}$ Eisessig und $10 \mathrm{ml} 48 \%$ $\mathrm{HBr} 2$ Stdn. am Rückfluß erhitzt. Nach dem Abkühlen wurde mit ca. $500 \mathrm{ml}$ Wasser verdünnt und zweimal mit je $200 \mathrm{ml}$ Essigester ausgeschüttelt. Der nach Eindampfen der über $\mathrm{Na}_{2} \mathrm{SO}_{4}$ getrockneten Extrakte verbleibende Rückstand wurde mit $40 \mathrm{ml}$ Acetanhydrid und einer Spur konz. Schwefelsäure wie oben beschrieben acetyliert. Nach dreimaligem Umkristallisieren aus Eisessig $350 \mathrm{mg}$, in allen Eigenschaften identisch mit dem aus natürlicher Xerocomsäure erhaltenen Produkt. Der Mischschmp. zeigte keine Depression.

\section{c) Xerocomsäure (3abzw. $\mathbf{3} b)$}

$20 \mathrm{mg} 6$ wurden in Essigester gelöst und auf eine $\mathrm{Al}_{2} \mathrm{O}_{3}$-Säule gegeben. Eluieren mit Methanol/konz. $\mathrm{HCl}$ (100:2) lieferte $5 \mathrm{mg}$ reine Xerocomsäure. Nach UVund Massenspektrum identisch mit dem Naturprodukt.

\subsection{4-Trihydroxy-anthrachinon-5-(und 8) carbonsäuren (1 $a$ und 1 b)}

Das Gemisch der beiden Verbindungen wurde nach KöGL und DeIJs ${ }^{12}$ dargestellt. Es konnte dünnschichtchromatographisch auf Kieselgel nicht aufgetrennt werden. Im Massenspektrum traten neben dem Molekularion $\mathrm{m} / \mathrm{e} 300$ markante Fragmentionen bei $\mathrm{m} / \mathrm{e} 282$ (M-18) und 254 (M-46) auf.

UV-Spektrum: $\lambda_{\max }=467,490$ und $520 \mathrm{~nm}$ (in Äthanol) ; $518,549 \mathrm{~nm}$ (in verd. $\mathrm{NaOH}$, wie l. c. ${ }^{12}$ ) und 466, 508 und $536 \mathrm{~nm}$ nach Zugabe von Kartoffelpreßsaft. Mit $\mathrm{K}_{3}\left[\mathrm{Fe}(\mathrm{CN})_{6}\right] / \mathrm{NaHCO}_{3}$ trat rasch Entfärbung ein, ebenso nach Versetzen der mit Bleitetraacetat behandelten Eisessiglösung mit wäßrigem Natriumhydrogencarbonat.

Dem Fonds der Chemischen Industrie danken wir für ein Stipendium für W. F., Der Stiftung Volkswagenwerk für die Mittel zur Beschaffung des Massenspektrometers MS 9. Dem Leibniz-Rechenzentrum der Bayerischen Akademie der Wissenschaften sind wir für die Überlassung von Rechenzeit zu Dank verpflichtet. 